\title{
Understanding Discourse on Work and Job-Related Well-Being in Public Social Media
}

\author{
Tong Liu ${ }^{1 *}$, Christopher M. Homan ${ }^{1 *}$, Cecilia Ovesdotter Alm ${ }^{1 \$}$, \\ Ann Marie White ${ }^{2}$, Megan C. Lytle ${ }^{2}$, Henry A. Kautz ${ }^{3}$ \\ 1* Golisano College of Computing and Information Sciences, Rochester Institute of Technology \\ $1 \$$ College of Liberal Arts, Rochester Institute of Technology \\ ${ }^{2}$ University of Rochester Medical Center \\ ${ }^{3}$ Department of Computer Science, University of Rochester \\ t18313@rit.edu, cmh@cs.rit.edu, coagla@rit.edu \\ annmarie_white|megan_lytledurmc.rochester.edu \\ kautz@cs.rochester.edu
}

\begin{abstract}
We construct a humans-in-the-loop supervised learning framework that integrates crowdsourcing feedback and local knowledge to detect job-related tweets from individual and business accounts. Using data-driven ethnography, we examine discourse about work by fusing languagebased analysis with temporal, geospational, and labor statistics information.
\end{abstract}

\section{Introduction}

Work plays a major role in nearly every facet of our lives. Negative and positive experiences at work places can have significant social and personal impacts. Employment condition is an important social determinant of health. But how exactly do jobs influence our lives, particularly with respect to well-being? Many theories address this question (Archambault and Grudin, 2012; Schaufeli and Bakker, 2004), but they are hard to validate as well-being is influenced by many factors, including geography as well as social and institutional support.

Can computers help us understand the complex relationship between work and well-being? Both are broad concepts that are difficult to capture objectively (for instance, the unemployment rate as a statistic is continually redefined) and thus challenging subjects for computational research.

Our first contribution is to propose a classification framework for such broad concepts as work that alternates between humans-in-the-loop annotation and machine learning over multiple iterations to simultaneously clarify human understanding of these concepts and automatically determine whether or not posts from public social media sites are about work. Our framework balances the effectiveness of crowdsourced workers with local experience, evaluates the degree of subjectivity throughout the process, and uses an iterative posthoc evaluation method to address the problem of discovering gold standard data. Our performance (on an open-domain problem) demonstrates the value of our humans-in-the-loop approach which may be of special relevance to those interested in discourse understanding, particularly settings characterized by high levels of subjectivity, where integrating human intelligence into active learning processes is essential.

Our second contribution is to use our classifiers to study job-related discourse on social media using data-driven ethnography. Language is fundamentally a social phenomenon, and social media gives us a lens through which to observe a very particular form of discourse in real time. We add depth to the NLP analysis by gathering data from specific geographical regions to study discourse along a broad spectrum of interacting social groups, using work as a framing device, and we fuse language-based analysis with temporal, geospatial and labor statistics dimensions.

\section{Background and Related Work}

Though not the first study of job-related social media, prior ones used data from large companies' internal sites, whose users were employees (De Choudhury and Counts, 2013; Yardi et al., 2008; Kolari et al., 2007; Brzozowski, 2009). An obvious limitation in that case is it excludes populations without access to such restricted networks. Moreover, workers may not disclose true feelings about their jobs on such sites, since their employ- 
ers can easily monitor them. On the other hand, we show that on Twitter, it is quite common for tweets to relate negative feelings about work ("I don't wanna go to work today"), unprofessional behavior ("Got drunk as hell last night and still made it to work"), or a desire to work elsewhere ("I want to go work at Disney World so bad").

Nonetheless, these studies inform our work. DeChoudhury et al. (2013) investigated the landscape of emotional expression of the employees via enterprise internal microblogging. Yardi et al. (2008) examined temporal aspects of blogging usage within corporate internal blogging community. Kolari et al. (2007) characterized comprehensively how behaviors expressed in posts impact a company's internal social networks. Brzozowski (2009) described a tool that aggregated shared internal social media which when combined with its enterprise directory added understanding the organization and employees connections.

From a theoretical perspective, the Job Demands-Resources Model (Schaufeli and Bakker, 2004) suggests that job demands (e.g., overworked, dissonance, and conflict) lead to burnout and disengagement while resources (e.g., appreciation, cohesion, and safety) often result in satisfaction and productivity. Although burnout and engagement have an inverse relationship, these states fluctuate and can vary over time. In 2014, more than two-thirds of U.S. workers were disengaged at work (Gallup, 2015a) and this disconnection costs the U.S. up to $\$ 398$ billion annually in lost work and medical treatment (Gallup, 2015b). Indeed, job dissatisfaction poses serious health risks and has even been linked to suicide (Hazards Magazine, 2014). Thus, examining social media for job-related messages provides a novel opportunity to study job discourse and associated demands and resources. Moreover, the declarative and affective tone of these tweets may have important implications for understanding the relationship between burnout and engagement with such public health concerns as mental health.

\section{Humans-in-the-Loop Classification}

From July 2013 to June 2014 we collected over $7 \mathrm{M}$ geo-tagged tweets from around 85,000 public accounts in a 15-county around a midsized city using DataSift ${ }^{1}$. We removed punctuation and special characters, and used the Internet Slang Dictio-

\footnotetext{
${ }^{1}$ http://datasift.com/
}

nary $^{2}$ to normalize nonstandard terms.

Figure 1 shows our humans-in-the-loop framework for learning classifiers to identify job-related posts. It consists of four rounds of machine classification - similar to that of Li et al. (2014) except that our rounds are not as uniform - where the classifier in each round acts as a filter on our training data, providing human annotators a sample of Twitter data to label and (except for the final round) using these labeled data to train the classifiers in later rounds.

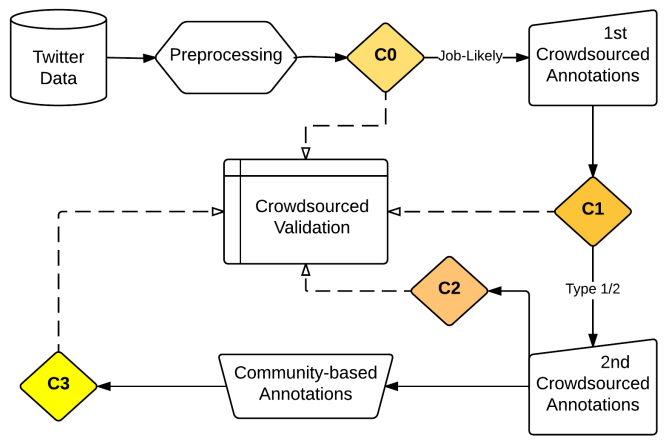

Figure 1: Flowchart of our humans-in-the-loop framework, laid out in Section 3.

The initial classifier $C_{0}$ is a simple termmatching filter; see Table 1 (number options were considered for some terms). The other classifiers $\left(C_{1}, C_{2}, C_{3}\right)$ are SVMs that use a feature space of n-grams from the training set.

\begin{tabular}{c|c} 
Include & $\begin{array}{c}\text { job, jobless, manager, boss } \\
\text { my/your/his/her/their/at work }\end{array}$ \\
\hline Exclude & $\begin{array}{c}\text { school, class, homework, student, course } \\
\text { finals, good/nice/great job, boss ass }{ }^{3}\end{array}$
\end{tabular}

Table 1: $C_{0}$ rules identifying Job-Likely tweets.

Round 1. We ran $C_{0}$ on our dataset. Approximately $40 \mathrm{~K}$ tweets having at least five tokens passed this filter. We call them Job-Likely tweets. We randomly chose around 2,000 JobLikely tweets and split them evenly into 50 AMT Human Intelligence Tasks (HITs), and further randomly duplicated five tweets in each HIT to evaluate each worker's consistency. Five crowdworkers assigned to each HIT $^{4}$ answered, for each tweet,

\footnotetext{
${ }^{2}$ http: //www. noslang.com/dictionary

${ }^{3}$ Describe something awesome in a sense of utter dominance, magical superiority, or being ridiculously good.

${ }^{4}$ This is based on empirical insights for crowdsourced annotation tasks (Callison-Burch, 2009; Evanini et al., 2010).
} 
the question: Is this tweet about job or employment? All crowdworkers lived in the U.S. and had an approval rating of $90 \%$ or better. They were paid $\$ 1.00$ per HIT $^{5}$. We assessed inter-annotator reliability among the five annotators in each HIT using Geertzen's tool (Geertzen, 2016).

This yielded 1,297 tweets where all 5 annotators agreed on the same label (Table 2). To balance our training data, we added 757 tweets chosen randomly from tweets outside the Job-Likely set that we labeled not job-related. $C_{1}$ trained on this set.

Round 2. Our goal was to collect 4,000 more labeled tweets that, when combined with the Round 1 training data, would yield a class-balanced set. Using $C_{1}$ to perform regression, we ranked the tweets in our dataset by the confidence score (Chang and Lin, 2011). We then spot-checked the tweets to estimate the frequency of job-related tweets as the confidence score increases. We discovered that among the top-ranked tweets about half, and near the separating hyperplane (i.e., where the confidence scores are near zero) almost none, are job-related.

Based on these estimates, we randomly sampled 2,400 tweets from those in the top 80th percentile of confidence scores (Type-1). We then randomly sampled about 800 tweets each from the first deciles of tweets greater and lesser than zero, respectively (Type-2).

The rationale for drawing from these two groups was that the false Type- 1 tweets represent those on which the $C_{1}$ classifier most egregiously fails, and the Type- 2 tweets are those closest to the feature vectors and those toward which the classifier is most sensitive.

Crowdworkers again annotated these tweets in the same fashion as in Round 1 (see Table 3), and cross-round comparisons are in Tables 2 and 4 . We trained $C_{2}$ on all tweets from Round 1 and 2 with unanimous labels (bold in Table 2).

\begin{tabular}{c|ccc|ccc}
\multirow{2}{*}{ AMTs } & \multicolumn{3}{|c|}{ job-related } & \multicolumn{3}{c}{ not job-related } \\
& $\mathbf{3}$ & $\mathbf{4}$ & $\mathbf{5}$ & $\mathbf{3}$ & $\mathbf{4}$ & $\mathbf{5}$ \\
\hline Round 1 & 104 & 389 & $\mathbf{1 0 2 7}$ & 78 & 116 & $\mathbf{2 7 0}$ \\
Round 2 & 140 & 287 & $\mathbf{7 2 1}$ & 66 & 216 & $\mathbf{2 5 6 8}$
\end{tabular}

Table 2: Summary of both annotation rounds.

\footnotetext{
${ }^{5}$ We consulted with Turker Nation (http://www. turkernation.com) to ensure that the workers were treated and compensated fairly for their tasks. We also rewarded annotators based on the qualities of their work.
}

\begin{tabular}{c|ccc|ccc}
\multirow{2}{*}{ Round 2 } & \multicolumn{3}{|c|}{ job-related } & \multicolumn{3}{c}{ not job-related } \\
& $\mathbf{3}$ & $\mathbf{4}$ & $\mathbf{5}$ & $\mathbf{3}$ & $\mathbf{4}$ & $\mathbf{5}$ \\
\hline Type-1 & 129 & 280 & 713 & 50 & 149 & 1079 \\
Type-2 & 11 & 7 & 8 & 16 & 67 & 1489
\end{tabular}

Table 3: Summary of tweet labels in Round 2 by confidence type (showing when $3 / 4 / 5$ of 5 annotators agreed).

\begin{tabular}{c|cc} 
AMTs & Fleiss' kappa & Krippendorf's alpha \\
\hline Round 1 & $0.62 \pm 0.14$ & $0.62 \pm 0.14$ \\
Round 2 & $0.81 \pm 0.09$ & $0.81 \pm 0.08$
\end{tabular}

Table 4: Average \pm stdev agreement from Round 1 and 2 are Good, Very Good (Altman, 1991).

\begin{tabular}{c|c} 
Annotations & Sample Tweet \\
\hline Y Y Y Y Y & $\begin{array}{c}\text { Really bored....., no entertainment } \\
\text { at work today }\end{array}$ \\
\hline$\underline{\text { Y Y Y Y N }}$ & $\begin{array}{c}\text { two more days of work then } \\
\text { I finally get a day off. }\end{array}$ \\
\hline$\underline{\text { Y Y Y N N }}$ & $\begin{array}{c}\text { Leaving work at 430 and } \\
\text { driving in this snow is going } \\
\text { to be the death of me }\end{array}$ \\
\hline Y Y N N N & $\begin{array}{c}\text { Being a mommy is the hardest } \\
\text { but most rewarding job } \\
\text { a women can have } \\
\text { \#babyBliss \#babybliss }\end{array}$ \\
\hline$\underline{\text { Y N N N N }}$ & $\begin{array}{c}\text { These refs need to } \\
\text { DO THEIR FUCKING JOBS }\end{array}$ \\
\hline N N N N N & $\begin{array}{c}\text { One of the best Friday nights } \\
\text { I've had in a while }\end{array}$
\end{tabular}

Table 5: Inter-annotator agreement combinations with sample tweets. Y denotes job-related. Cases where the majority (not all) annotators agreed (3/4 out of 5) are underlined in bold.

Round 3. Two coauthors with prior experience from the local community reviewed instances from Round 1 and 2 on which crowdworkers disagreed (highlighted in Table 5) and provided labels. Cohen's kappa agreement was high: $\kappa=0.80$. Combined with all labeled data from the previous rounds this yielded 2,670 goldstandard-labeled job-related and 3,250 not jobrelated tweets. We trained $C_{3}$ on this entire set. Since it is not strictly class-balanced, we gridsearched on a range of class weights and chose the estimator that optimized F1 score, using 10fold cross validation ${ }^{6}$. Table 6 shows $C_{3}$ 's topweighted features, which reflect the semantic field of work for the job-related class.

\footnotetext{
${ }^{6}$ These scores were determined respectively using the mean score over the cross-validation folds. The parameter settings that gave the best results on the left out data were a linear kernel with penalty parameter $C=0.1$ and class weight ratio of $1: 1$.
} 


\begin{tabular}{cc|cc} 
job-related & weights & not job-related & weights \\
\hline work & 2.026 & did & -0.714 \\
job & 1.930 & amazing & -0.613 \\
manager & 1.714 & nut & -0.600 \\
jobs & 1.633 & hard & -0.571 \\
managers & 1.190 & constr & -0.470 \\
working & 0.827 & phone & -0.403 \\
bosses & 0.500 & doing & -0.403 \\
lovemyjob & 0.500 & since & -0.373 \\
shift & 0.487 & brdg & -0.363 \\
worked & 0.410 & play & -0.348 \\
paid & 0.374 & its & -0.337 \\
worries & 0.369 & think & -0.330 \\
boss & 0.369 & thru & -0.329 \\
seriously & 0.368 & hand & -0.321 \\
money & 0.319 & awesome & -0.319
\end{tabular}

Table 6: Top 15 features for both classes of $C_{3}$.

Discovering Businesses. Manual examination of job-related tweets revealed patterns like: Panera Bread: Baker - Night (\#LOCATION) http://URL \#Hospitality \#VeteranJob \#Job \#Jobs \#TweetMyJobs. Nearly all tweets that contained at least one of these hashtags: \#veteranjob, \#job, \#jobs, \#tweetmyjobs, \#hiring, \#retail, \#realestate, $\# h r$ also included a URL, which spot-checking revealed nearly always led to a recruitment website (see Table 7). This led to an effective heuristic to separate individual from business accounts only for posts that have first been classified as jobrelated: if an account had more job-related tweets with any of the above hashtags + URL patterns, we labeled it business; otherwise individual.

\begin{tabular}{c|cc} 
& hashtag only & hashtag + URL \\
\hline \#veteranjob & 18,066 & 18,066 \\
\#job & 79,362 & 79,327 \\
\#jobs & 58,637 & 58,631 \\
\#tweetmyjobs & 39,007 & 39,007 \\
\#hiring & 148 & 147 \\
\#retail & 17,037 & 17,035 \\
\#realestate & 92 & 92 \\
\#hr & 400 & 399
\end{tabular}

Table 7: Counts of hashtags queried, and counts of their subsets with hashtags coupled with URL.

\section{Results and Discussion}

Crowdsourced Validation The fundamental difficulty in open-domain classification problems such as this one is there is no gold-standard data to hold out at the beginning of the process. To address this, we adopted a post-hoc evaluation where we took balanced sets of labeled tweets from each classifier $\left(C_{0}, C_{1}, C_{2}\right.$ and $\left.C_{3}\right)$ and asked AMT workers to label a total of 1,600 sam- ples, taking the majority votes (where at least 3 out of 5 crowdworkers agreed) as reference labels. Our results (Table 8) show that $C_{3}$ performs the best, and significantly better than $C_{0}$ and $C_{1}$.

Estimating Effective Recall The two machinelabeled classes in our test data are roughly balanced, which is not the case in real-world scenarios. We estimated the effective recall under the assumption that the error rates in our test samples are representative of the entire dataset. Let $y$ be the total number of the classifier-labeled "positive" elements in the entire dataset and $n$ be the total of "negative" elements. Let $y_{t}$ be the number of classifier-labeled "positive" tweets in our 1,600samples test set and let $n_{t}=1,600-y_{t}$. Then the estimated effective recall $\hat{R}=\frac{y \cdot n_{t} \cdot R}{y \cdot n_{t} \cdot R+n \cdot y_{t} \cdot(1-R)}$.

\begin{tabular}{cc|cccc} 
Model & Class & $\mathbf{P}$ & $\mathbf{R}$ & $\hat{\mathbf{R}}$ & $\mathbf{F 1}$ \\
\hline \multirow{2}{*}{$C_{0}$} & job & 0.72 & 0.33 & 0.01 & 0.45 \\
& notjob & 0.68 & 0.92 & 1.00 & 0.78 \\
\hline \multirow{2}{*}{$C_{1}$} & job & 0.79 & 0.82 & 0.15 & 0.80 \\
& notjob & 0.88 & 0.86 & 0.99 & 0.87 \\
\hline \multirow{2}{*}{$C_{2}$} & job & 0.82 & 0.95 & 0.41 & 0.88 \\
& notjob & 0.97 & 0.86 & 0.99 & 0.91 \\
\hline \multirow{2}{*}{$C_{3}$} & job & 0.83 & 0.96 & 0.45 & 0.89 \\
& notjob & 0.97 & 0.87 & 0.99 & 0.92
\end{tabular}

Table 8: Crowdsourced validations of instances identified by 4 distinct models (1,600 total tweets).

Assessing Business Classifier For Table 8's tweets labeled by $C_{0}-C_{3}$ as job-related, we asked AMT workers: Is this tweet more likely from a personal or business account? Table 9 shows that this method was quite accurate.

\begin{tabular}{cc|ccc} 
From & Class & $\mathbf{P}$ & $\mathbf{R}$ & F1 \\
\hline \multirow{3}{*}{$C_{0}$} & individual & 0.86 & 1.00 & 0.92 \\
& business & 0.00 & 0.00 & 0.00 \\
& avg/total & 0.74 & 0.86 & 0.79 \\
\hline \multirow{3}{*}{$C_{1}$} & individual & 1.00 & 0.97 & 0.98 \\
& business & 0.98 & 1.00 & 0.99 \\
& avg/total & 0.99 & 0.99 & 0.99 \\
\hline \multirow{2}{*}{$C_{2}$} & individual & 1.00 & 0.98 & 0.99 \\
& business & 0.98 & 1.00 & 0.99 \\
& avg/total & 0.99 & 0.99 & 0.99 \\
\hline \multirow{2}{*}{$C_{3}$} & individual & 1.00 & 0.99 & 0.99 \\
& business & 0.99 & 1.00 & 0.99 \\
& avg/total & 0.99 & 0.99 & 0.99
\end{tabular}

Table 9: Crowdsourced validations of individuals vs. businesses job-related tweets.

Our explanation for the strong performance of the business classifier is that the class of jobrelated tweets is relatively rare, and so by applying the classifier only to job-related tweets we sim- 
plify the individual-or-business problem dramatically. Another, perhaps equally effective, simplification is that our tweets are geo-specific and so we automatically filter out business tweets from, e.g., national media.

Generalizability Tests Can our best model $C_{3}$ discover job-related tweets from other geographical regions, even though it was trained on data from one specific region? We repeated the tests above on 400 geo-tagged tweets from Detroit (balanced between job-related and not). Table 10 shows that $C_{3}$ and the business classifier generalize well to another region. This suggests the transferability of our humans-in-the-loop classification framework and of heuristic to separate individual from business accounts for tweets classified as job-related.

\begin{tabular}{cc|c|c|c} 
Model & Class & P & R & F1 \\
\hline \multirow{2}{*}{$C_{3}$} & job & 0.85 & 0.99 & 0.92 \\
& notjob & 0.99 & 0.87 & 0.93 \\
\hline \multirow{3}{*}{ Heuristic } & individual & 1.00 & 0.96 & 0.98 \\
& business & 0.96 & 1.00 & 0.98 \\
& avg/total & 0.98 & 0.98 & 0.98
\end{tabular}

Table 10: Validations of $C_{3}$ and business classifier on Detroit data.

\section{Understanding Job-Related Discourse}

Using the job-related tweets - from both individual and business accounts - extracted by $C_{3}$ from the July 2013-June 2014 dataset (see Table 12), we conducted the following analyses.

$\mathbf{C}_{3}$ Versus $\mathbf{C}_{\mathbf{0}}$ The fact that $C_{3}$ outperforms $C_{0}$ demonstrates our humans-in-the-loop framework is necessary and effective compared to an intuitive term-matching filter. We further examined the messages labeled as job-related by $C_{3}$, but not captured by $C_{0}$. More than 160,000 tweets fell into this Difference set, in which approximately 85,000 tweets are from individual accounts while the rest are from business accounts. Table 11 shows the top 3 most frequent uni-, bi-, and trigrams in the Difference dataset. These n-grams from the individual group suggest that people often talk about job-related topics while mentioning temporal information or announcing their working schedules. We neglected such time-related phrases when defining $C_{0}$. In contrast, the frequencies of the listed n-grams in the business group are much higher than those in the individual group. This indicates that our definitions of inclusion terms in
$C_{0}$ did not capture a considerable amount of posts involving broad job-related topics, which is also reflected in Table 9: our business classifier did not find business accounts from the job-related tweets extracted by $C_{0}$.

\begin{tabular}{c|c} 
Individual & Business \\
\hline \multicolumn{2}{|c}{ Unigrams } \\
\hline day, 6989 & ny, 83907 \\
\hline today, 5370 & \#job, 75178 \\
\hline good, 4245 & \#jobs, 55105 \\
\hline \multicolumn{2}{|c}{ Trigrams } \\
\hline last night, 359 & \#jobs \#tweetmyjobs, 32165 \\
\hline getting ready, 354 & \#rochester ny, 22101 \\
\hline first day, 296 & \#job \#jobs, 16923 \\
\hline working hour & \#job \#jobs \\
shift, 51 & \#tweetmyjobs, 12004 \\
\hline first day & ny \#jobs \\
back, 48 & \#tweetmyjobs, 4955 \\
\hline separate leader & ny \#retail \\
follower, 44 & \#job, 4704
\end{tabular}

Table 11: Top 3 most frequent uni-, bi-, and trigrams with frequencies in the Difference set.

Hashtags Individuals posted 11,935 unique hashtags and businesses only 414 . The top 250 hashtags from each group are shown in Figure 2.

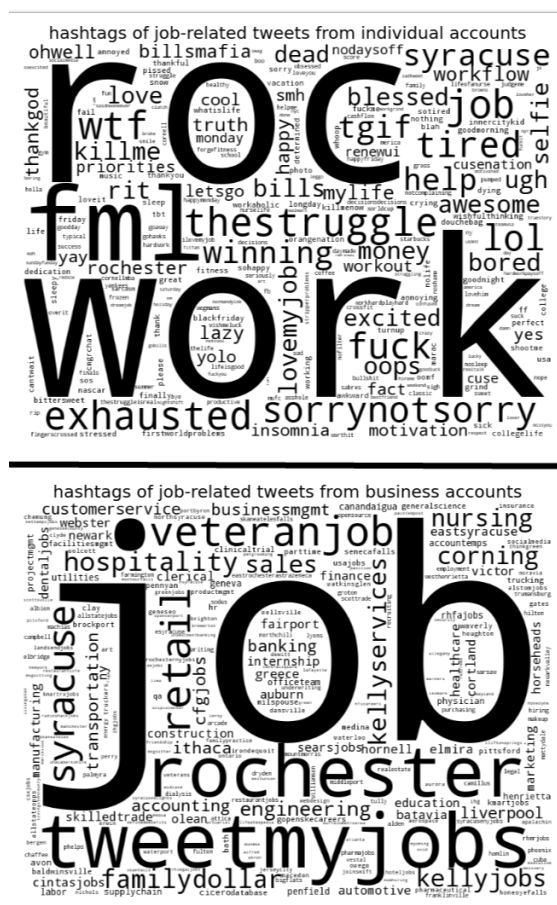

Figure 2: Hashtags in job-related tweets: above individual accounts; below - business accounts.

Individual users used an abbreviation for the name of the midsized city to mark their location, 
and $\mathrm{fml}^{7}$ to express personal embarrassing stories. Work and job are self-explanatory. Money, motivation relates to jobs. Tired, exhausted, fuck, insomnia, bored, struggle express negative conditions. Likewise, lovemyjob, happy, awesome, excited, yay, tgif $^{8}$ convey positive affects experienced from jobs. Business accounts exhibit distinct patterns. Besides the hashtags queried (Table 7), we saw local place names, like corning, rochester, batavia, pittsford, and regional ones like syracuse, ithaca. Customerservice, nursing, accounting, engineering, hospitality, construction record occupations, while kellyjobs, familydollar, cintasjobs, cfgjobs, searsjobs point to business agents. Unlike individual users, businesses do not use hashtags reflecting affective expressions.

Linguistic Differences We used the TweetNLP POS tagger (Gimpel et al., 2011). Figure 3 shows nine part-of-speech tag ${ }^{9}$ frequencies for three subsets of tweets.

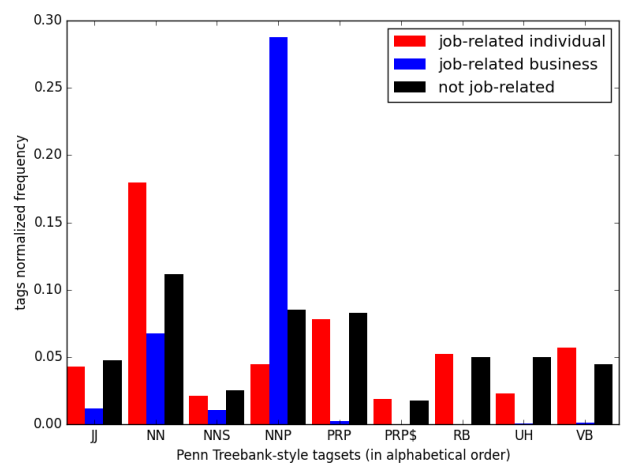

Figure 3: POS tag comparisons (normalized, averaged) among three subsets of tweets: job-related tweets from individual accounts (red), job-related tweets from business accounts (blue) and not jobrelated tweets (black).

Business accounts use NNPs more than individuals, perhaps because they often advertise job openings at specific locations, like New York, Sears. Individuals use NNPs less frequently and in a more casual way, e.g., Jojo, galactica, Valli. Also, individuals use JJ, NN, NNS, PRP, PRP\$, $\mathrm{RB}, \mathrm{UH}$, and VB more regularly than business ac-

\footnotetext{
${ }^{7}$ An acronym for Fuck My Life.

${ }^{8}$ An acronym for Thank God It's Friday to express the joy one feels in knowing that the work week has officially ended and that one has two days off which to enjoy.

${ }^{9} \mathrm{JJ}$ - Adjective; NN - Noun (singular or mass); NNS Noun (plural); NNP - Proper noun (singular); PRP - Personal pronoun; PRP\$ - Possessive pronoun; RB - Adverb; UH Interjection; VB - Verb (base form) (Santorini, 1990).
}

counts do. Not job-related tweets have similar patterns to job-related ones from individual accounts, suggesting that individual users exhibit analogous language habits regardless of topic.

Temporal Patterns Our findings that individual users frequently used time-related n-grams (Table 11) prompted us to examine the temporal patterns of job discourse.

Figure 4a suggests that individuals talk about jobs the most in December and January (which also have the most tweets over other topics), and the least in the warmer months. July witnesses the busiest job-related tweeting from business and January the least. The user community is slightly less active in the warmer months, with fewer tweets then.

Figure $4 \mathrm{~b}$ shows that job-related tweet volumes are higher on weekdays and lower on weekends, following the standard work week. Weekends see fewer business tweets than weekdays do. Sunday is the most - while Friday and Saturday are the least - active days from the not job-related perspective.

Figure $4 \mathrm{c}$ shows hourly trends. Job-related tweets from business accounts are most frequent during business hours, peaking at 11 , and then taper off. Perhaps professionals are either getting their commercial tasks completed before lunch, or expecting others to check updates during lunch. Individuals post about jobs almost anytime awake and have a similar distribution to non-job-related tweets.

Measuring Affective Changes We examined positive affect (PA) and negative affect (NA) to measure diurnal changes in public mood (Figures 5 and 6), using two recognized lexicons, in jobrelated tweets from individual accounts (left), jobrelated tweets from business accounts (middle), and not job-related tweets (right).

(1) Linguistic Inquiry and Word Count We used LIWC's positive emotion and negative emotion to represent PA and NA respectively (Pennebaker et al., 2001) because it is common in behavioral health studies, and used as a standard comparison in referenced work. Figure 5 shows the mean daily trends of PA and NA. ${ }^{10}$ Panels $5 \mathrm{a}$ and $5 \mathrm{~b}$ reveal contrasting job-related affective patterns, compared to prior trends from

\footnotetext{
${ }^{10}$ Non-equal y-axes help show peak/valley patterns here and in Figure 6, also motivated by lexicon's unequal sizes.
} 


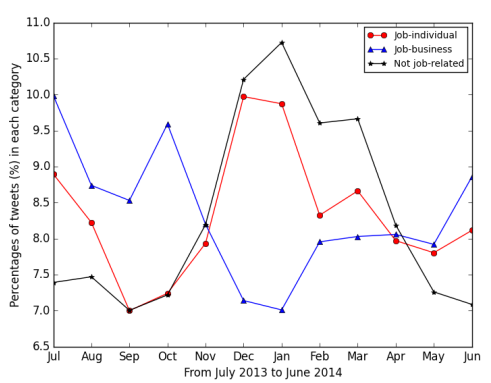

(a) In each month

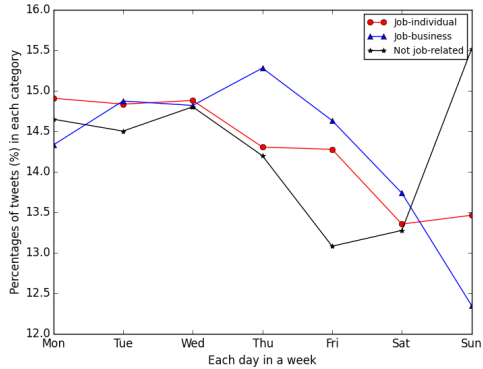

(b) On each day of week

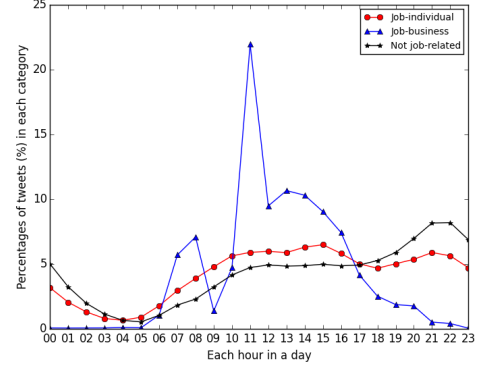

(c) In each hour

Figure 4: Distributions of job-related tweets over time by job class. We converted timestamps from the Coordinated Universal Time standard (UTC) to local time zone with daylight saving time taken into account.

enterprise-wide micro-blog usage (De Choudhury and Counts, 2013), i.e., public social media exhibit gradual increase in PA while internal enterprise network decrease after business. This perhaps confirms our suspicion that people talk about work on public social media differently than on work-based media.

(2) Word-Emotion Association Lexicon We focused on the words from EmoLex's positive and negative categories, which represent sentiment polarities (Mohammad and Turney, 2013; Mohammad and Turney, 2010) and calculated the score for each tweet similarly as LIWC. The average daily positive and negative sentiment scores in Figure 6 display patterns analogous to Figure 5.

Labor Statistics We explored associations between Twitter temporal patterns, affect, and official labor statistics (Figure 8). These monthly statistics ${ }^{11}$ include: labor force, employment, unemployment, and unemployment rate. We collected one more year of Twitter data from the same area, and applied $C_{3}$ to extract the jobrelated posts from individual and business accounts (Table 12 summarizes the basic statistics), then defined the following monthwise statistics for our two-year dataset: count of overall/jobindividual/job-business/others tweets; percentage of job-individual/job-business/others tweets in overall tweets; average LIWC PA/NA scores of job-individual/job-business/others tweets ${ }^{12}$.

Positive affect expressed in job-related discourse from both individual and business accounts correlate negatively with unemployment and un-

\footnotetext{
${ }^{11}$ Published by US Department of Labor, including: Local Area Unemployment Statistics; State and Metro Area Employment, Hours, and Earnings.

${ }^{12}$ IND: individual; BIZ: business; pct: \%; avg: average.
}

employment rate. This is intuitive, as unemployment is generally believed to have a negative impact on individuals' lives. The counts of jobrelated tweets from individual and not job-related tweets are both positively correlated with unemployment and unemployment rate, suggesting that unemployment may lead to more activities in public social media. This correlation result shows that online textual disclosure themes and behaviors can reflect institutional survey data.

Inside vs. Outside City We compared tweets occurring within the city boundary to those lying outside (Table 13). The percentages of job-related tweets from individual accounts, either in urban or rural areas, remain relatively even. The proportion of job-related tweets from business accounts decreased sharply from urban to rural locations. This may be because business districts are usually centered in urban areas and individual tweets reflect more complex geospatial distributions.

Job-Life Cycle Model Based on hand inspection of a large number of job-related tweets and on models of the relationship between work and wellness found in behavioral studies (Archambault and Grudin, 2012; Schaufeli and Bakker, 2004), we tentatively propose a job-life model for jobrelated discourse from individual accounts (Figure 7). Each state in the model has three dimensions: the point of view, the affect, and the job-related activity, in terms of basic level of employment, expressed in the tweet.

We concatenated together all job-related tweets posted by each individual into a single document and performed latent Dirichlet allocation (LDA) (Blei et al., 2003) on this user-level corpus, using Gensim (̌̌ehưřek and Sojka, 2010). We used 12 


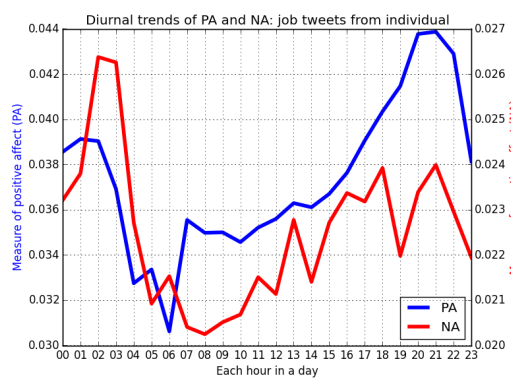

(a) Job-related tweets from individuals

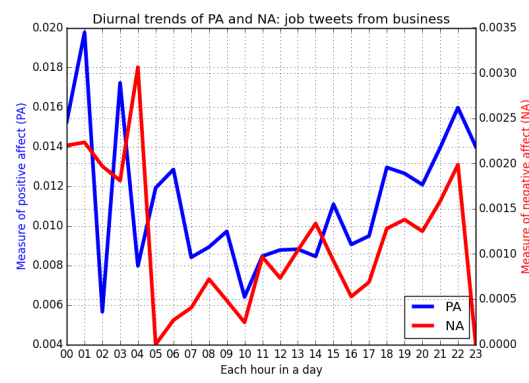

(b) Job-related tweets from businesses

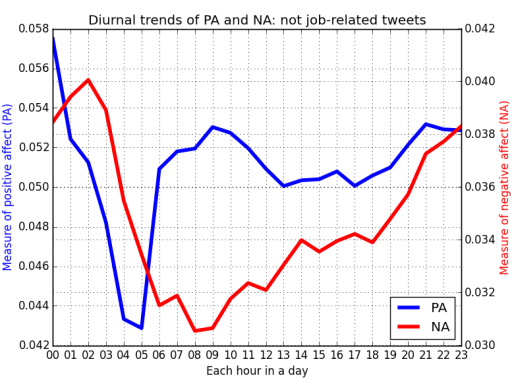

(c) Not job-related tweets

Figure 5: Diurnal trends of positive and negative affect based on LIWC.

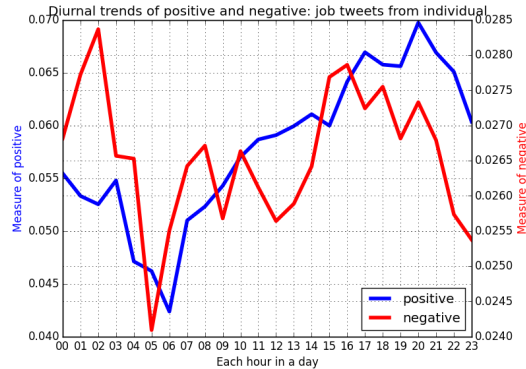

(a) Job-related tweets from individuals

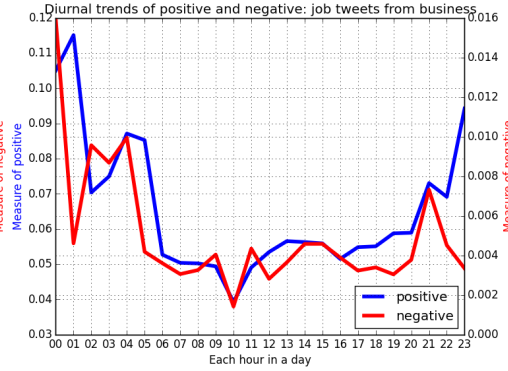

(b) Job-related tweets from businesses

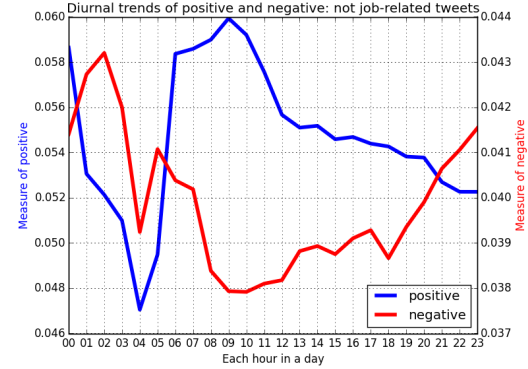

(c) Not job-related tweets

Figure 6: Diurnal trends of positive and negative affect based on EmoLex.

\begin{tabular}{|c|c|c|c|c|c|c|}
\hline \multirow[t]{2}{*}{ Unique counts } & \multirow{2}{*}{\multicolumn{2}{|c|}{$\begin{array}{l}\text { job-related tweets } \\
\text { from individual accounts } \\
\text { tweets accounts }\end{array}$}} & \multirow{2}{*}{\multicolumn{2}{|c|}{$\begin{array}{l}\text { job-related tweets } \\
\text { from business accounts }\end{array}$}} & \multicolumn{2}{|c|}{ not job-related tweets } \\
\hline & & & & & tweets & accounts \\
\hline July 20 & 114,302 & 17,183 & 79,721 & 292 & 306 & 718 \\
\hline July & 85,851 & & 115 , & 333 & 943 & 716 \\
\hline Total (unique counts) & 200,153 & 28,161 & 195,023 & 431 & $12,399,249$ & 136,703 \\
\hline
\end{tabular}

Table 12: Summary statistics of the two-year Twitter data classified by $C_{3}$.

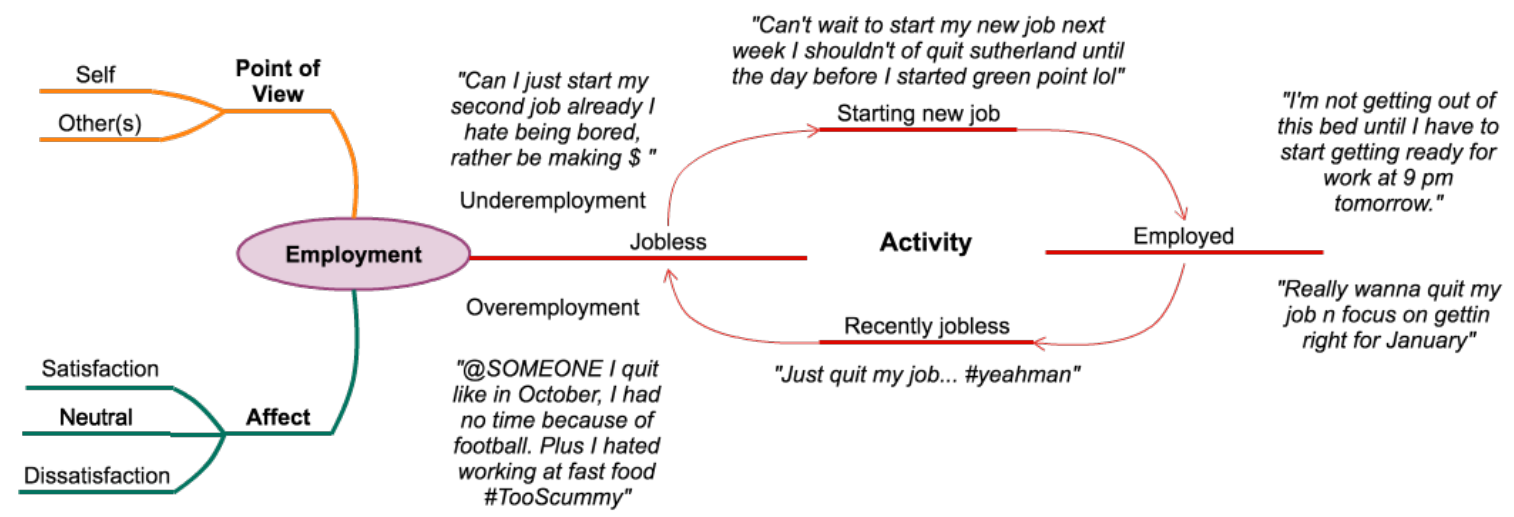

Figure 7: The job-life model captures the point of view, affect, and job-related activity in tweets.

\begin{tabular}{c|c|c|c}
$\%$ & $\begin{array}{c}\text { job-related } \\
\text { individual }\end{array}$ & $\begin{array}{c}\text { job-related } \\
\text { business }\end{array}$ & others \\
\hline Inside & 1.59 & 3.73 & 94.68 \\
$\begin{array}{c}\text { Outside } \\
\text { Combined }\end{array}$ & 1.85 & 1.51 & 96.65 \\
& 1.82 & 1.77 & 96.41
\end{tabular}

Table 13: Percent inside and outside city tweets. topics for the LDA based on the number of affect classes (three) times the number of job-related activities (four). See Table 14.

Topic 0 appears to be about getting ready to start a job, and topic 1 about leaving work permanently or temporarily. Topics 2, 5, 6, 8, and 11 suggest how key affect is for understanding job- 


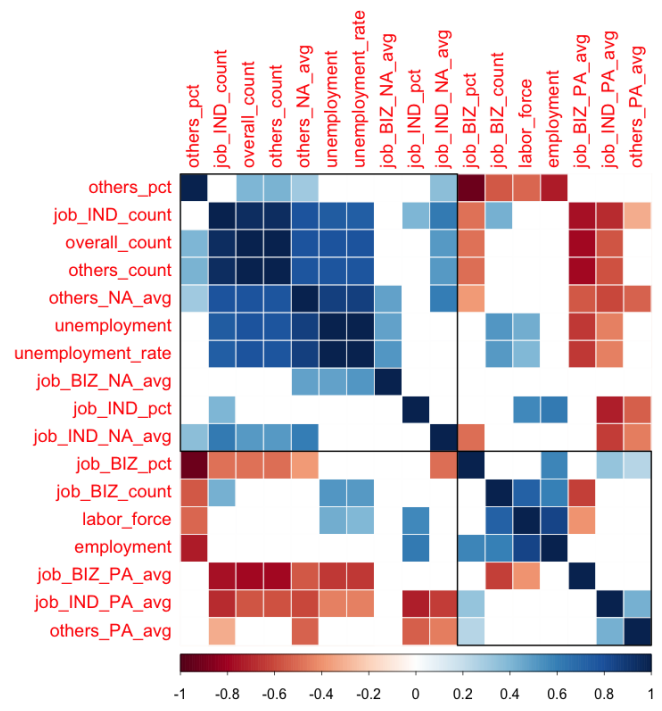

Figure 8: Correlation matrix with Spearman used for test at level .05 , with insignificant coefficients left blank. The matrix is ordered by a hierarchical clustering algorithm. Blue - positive correlation, red - negative correlation.

\begin{tabular}{c|c} 
Topic index & Representative words \\
\hline $\mathbf{0}$ & getting, ready, day, first, hopefully \\
$\mathbf{1}$ & last, finally, week, break, last_day \\
$\mathbf{2}$ & fucking, hate, seriously, lol, really \\
$\mathbf{3}$ & come_visit, some, talking, pissed \\
$\mathbf{4}$ & weekend, today, home, thank_god \\
$\mathbf{5}$ & wish, love, better, money, working \\
$\mathbf{6}$ & shift, morning, leave, shit, bored \\
$\mathbf{7}$ & manager, guy, girl, watch, keep \\
$\mathbf{8}$ & feel, sure, supposed, help, miss \\
$\mathbf{9}$ & much, early, long, coffee, care \\
$\mathbf{1 0}$ & time, still, hour, interview, since \\
$\mathbf{1 1}$ & best, pay, bored, suck, proud
\end{tabular}

Table 14: The top five words in each of the twelve topics discovered by LDA.

related discourse: 2 and 6 lean towards dissatisfaction and 5 toward satisfaction. 11 looks like a mixture. Topic 7 connects to coworkers. Many topics point to the importance of time (including leisure time in topic 4).

\section{Conclusion}

We used crowdsourcing and local expertise to power a humans-in-the-loop classification framework that iteratively improves identification of public job-related tweets. We separated business accounts from individual in job-related discourse. We also analyzed identified tweets integrating temporal, affective, geospatial, and statistical information. While jobs take up enormous amounts of most adults' time, job-related tweets are still rather infrequent. Examining affective changes reveals that PA and NA change independently; low NA appears to indicate the absence of negative feelings, not the presence of positive ones.

Our work is of social importance to workingage adults, especially for those who may struggle with job-related issues. Besides providing insights for discourse and its links to social science, our study could lead to practical applications, such as: aiding policy-makers with macro-level insights on job markets, connecting job-support resources to those in need, and facilitating the development of job recommendation systems.

This work has limitations. We did not study whether providing contextual information in our humans-in-the-loop framework would influence the model performance. This is left for future work. Additionally we recognize that the hashtag inventory used to discover business accounts from job-related topics might need to change over time, to achieve robust performance in the future. As another point, due to Twitter demographics, we are less likely to observe working seniors.

\section{Acknowledgments}

We thank the anonymous reviewers for their helpful comments and suggestions. This work was supported in part by a GCCIS Kodak Endowed Chair Fund Health Information Technology Strategic Initiative Grant and NSF Award \#SES-1111016.

\section{References}

DG Altman. 1991. Inter-Rater Agreement. Practical Statistics for Medical Research, 5:403-409.

Anne Archambault and Jonathan Grudin. 2012. A Longitudinal Study of Facebook, LinkedIn, \& Twitter Use. In Proceedings of the SIGCHI Conference on Human Factors in Computing Systems, pages 2741-2750. ACM.

David M Blei, Andrew Y Ng, and Michael I Jordan. 2003. Latent Dirichlet Allocation. the Journal of Machine Learning Research, 3:993-1022.

Michael J Brzozowski. 2009. Watercooler: Exploring an Organization through Enterprise Social Media. In Proceedings of the ACM 2009 International Conference on Supporting Group Work, pages 219228. ACM.

Chris Callison-Burch. 2009. Fast, Cheap, and Creative: Evaluating Translation Quality Using Amazon's Mechanical Turk. In Proceedings of the 2009 
Conference on Empirical Methods in Natural Language Processing: Volume 1-Volume 1, pages 286295. Association for Computational Linguistics.

Chih-Chung Chang and Chih-Jen Lin. 2011. LIBSVM: A Library for Support Vector Machines. ACM Transactions on Intelligent Systems and Technology (TIST), 2(3):27.

Munmun De Choudhury and Scott Counts. 2013. Understanding Affect in the Workplace via Social Media. In Proceedings of the 2013 Conference on Computer Supported Cooperative Work, pages 303-316. ACM.

Keelan Evanini, Derrick Higgins, and Klaus Zechner. 2010. Using Amazon Mechanical Turk for Transcription of Non-Native Speech. In Proceedings of the NAACL HLT 2010 Workshop on Creating Speech and Language Data with Amazon's Mechanical Turk, pages 53-56. Association for Computational Linguistics.

Gallup. 2015a. Majority of U.S. Employees not Engaged despite Gains in 2014.

Gallup. 2015b. Only 35\% of U.S. Managers are Engaged in their Jobs.

Jeroen Geertzen. 2016. Inter-Rater Agreement with Multiple Raters and Variables. [Online; accessed 17-February-2016].

Kevin Gimpel, Nathan Schneider, Brendan O'Connor, Dipanjan Das, Daniel Mills, Jacob Eisenstein, Michael Heilman, Dani Yogatama, Jeffrey Flanigan, and Noah A Smith. 2011. Part-of-Speech Tagging for Twitter: Annotation, Features, and Experiments. In Proceedings of the 49th Annual Meeting of the Association for Computational Linguistics: Human Language Technologies: short papersVolume 2, pages 42-47. Association for Computational Linguistics.

Hazards Magazine. 2014. Work Suicide.

Pranam Kolari, Tim Finin, Kelly Lyons, Yelena Yesha, Yaacov Yesha, Stephen Perelgut, and Jen Hawkins. 2007. On the Structure, Properties and Utility of Internal Corporate Blogs. Growth, 45000:50000.

Jiwei Li, Alan Ritter, Claire Cardie, and Eduard H Hovy. 2014. Major Life Event Extraction from Twitter based on Congratulations/Condolences Speech Acts. In EMNLP, pages 1997-2007.

Saif M Mohammad and Peter D Turney. 2010. Emotions Evoked by Common Words and Phrases: Using Mechanical Turk to Create An Emotion Lexicon. In Proceedings of the NAACL HLT 2010 Workshop on Computational Approaches to Analysis and Generation of Emotion in Text, pages 26-34. Association for Computational Linguistics.

Saif M. Mohammad and Peter D. Turney. 2013. Crowdsourcing a Word-Emotion Association Lexicon. 29(3):436-465.
James W Pennebaker, Martha E Francis, and Roger J Booth. 2001. Linguistic Inquiry and Word Count: LIWC 2001. Mahway: Lawrence Erlbaum Associates, 71:2001.

Radim Řehůřek and Petr Sojka. 2010. Software Framework for Topic Modelling with Large Corpora. In Proceedings of the LREC 2010 Workshop on New Challenges for NLP Frameworks, pages 4550, Valletta, Malta, May. ELRA.

Beatrice Santorini. 1990. Part-of-Speech Tagging Guidelines for the Penn Treebank Project (3rd Revision).

Wilmar B Schaufeli and Arnold B Bakker. 2004. Job Demands, Job Resources, and Their Relationship with Burnout and Engagement: A MultiSample Study. Journal of Organizational Behavior, 25(3):293-315.

Sarita Yardi, Scott Golder, and Mike Brzozowski. 2008. The Pulse of the Corporate Blogosphere. In Conf. Supplement of CSCW 2008, pages 8-12. Citeseer. 